\title{
Particle Filter Vehicle Tracking Based on SURF Feature Matching
}

\author{
Xiaofeng $\mathrm{Lu}^{*, * *}$ Student Member, Takashi Izumi ${ }^{* a)}$ Member \\ Lin Teng* $\quad$ Student Member, Lei Wang** ${ }^{* *} \quad$ Non-member
}

(Manuscript received May 15, 2013, revised Oct. 1, 2013)

\begin{abstract}
In this paper, we propose a robust vehicle tracking method based on speeded-up robust features (SURF) feature matching in a particle filter framework. In this framework, the color feature and the local binary pattern (LBP) texture feature are also combined to improve the representation of the tracking target. To further improve the tracking performance, three strategies are used. First, a dynamic update mechanism of the target template is proposed to capture appearance changes. Second, the size of the tracking window is also modified dynamically by balancing the weights of three feature distributions. Third, the weight of each particle is allocated with an improved distance kernel function method in the tracking process. Specifically, the proposed method of adopting new feature points for the target template can objectively reflect tracking target changes and effectively overcome the disadvantages of the random selection mechanism. We test the proposed approach on numerous sequences involving different types of challenges, including variations in illumination, scale changes, and rotation. The experimental results show that the proposed method is more efficient and robust than the classical approaches.
\end{abstract}

Keywords: particle filter, vehicle tracking, SURF, multiple features

\section{Introduction}

Visual surveillance in dynamic scenes has become a very important research area of image processing and computer vision techniques in recent years, which attempts to detect, recognize and track certain objects from image sequences, and more generally to understand and describe object behaviors ${ }^{(1)}$. The utilization of image-based traffic surveillance includes analysis the traffic flow and the status of road congestion, detection and avoidance of vehicle accidents, detection of vehicles with unusual behavior, and gathering of statistical information about roadway traffic. Vehicle tracking plays an important role in traffic surveillance because it provides dynamic information about the position of a tracked vehicle in time $^{(2)}$.

In this paper, we propose a robust vehicle tracking method based on speeded-up robust features (SURF) feature matching in a particle filter framework. In this framework, the color feature and the local binary pattern (LBP) texture feature are also combined to improve the representation of the tracking target. In our method, the following three mechanisms are proposed, such as, the dynamic update mechanism of target template, the modification mechanism of tracking window, and the allocation mechanism of particle weights. By comparing other state-of-the-art trackers through challenging sequences, the experimental results clearly demonstrate the

\footnotetext{
a) Correspondence to: Takashi Izumi. E-mail: izumi@ecs.cst. nihon-u.ac.jp

${ }^{*}$ Graduate School of Science and Technology, Nihon University

7-24-1, Narashinodai, Funabashi, Chiba 274-8501, Japan

** Faculty of Computer Science and Engineering, Xi' an University of Technology

5, South Jinhua Road, Xi' an Shaanxi 710048, China
}

effectiveness of our method.

Many different techniques have been proposed for tracking object in literatures. For example, in which Mean shift algorithm was proposed ${ }^{(7)}$, the feature histograms based on target representations are regularized by spatial masking with an isotropic kernel. The template of a target is achieved via detection and a cost function is established to describe the similarity between target candidate and template. According to the maximum of the cost function, target location is determined. Mean shift is quick and effective in some scenes. However, it cannot be adopted in targets with large scale variation.

A number of Bayesian stochastic filters have been used with success in object tracking applications. The most commonly used are Linear Kalman Filter (LKF) ${ }^{(8)(9)}$, Extended Kalman Filter (EKF) ${ }^{(10)}$, and Unscented Kalman Filter $(\mathrm{UKF})^{(11)}$. These filters have been utilized to relax the linearity assumption of the system model and thus to improve the tracking capabilities. However, these methods are limited to linear or Gaussian systems, and single modal probability distribution. In more complex scenes, the detectability of moving object will significantly reduce or track failure.

The particle filter methods have been introduced for object tracking in nonlinear, non-Gaussian, multi-modal situations. These algorithms are based on sequential Monte Carlo sampling methods and represent the required posterior density function by a set of random samples with associated weights and to compute an estimation based on these samples and weights ${ }^{(12)}$.

To represent target objects, a common solution is to use various features, such as color, edges, texture and motion. Color histograms have been widely used in the particle filter for likelihood estimation ${ }^{(3)-(5)}$. Color histograms are robust to 
partial occlusions, unrestrictive about the type of objects being tracked and can be computed efficiently. However, the main problem in tracking objects with a single color feature is ambiguous in scene with objects or regions with similar color properties to those of target objects. To improve the target model representation, a combination of features is commonly used. For instance, spatial information based on multiple color histograms computed on semi-overlapping image areas can be introduced ${ }^{(13)}$. The combination of shape and color is used ${ }^{(14)}$. The likelihood of each feature is calculated and weighted before Bayes' rule is applied to obtain the resultant posterior. Multiple multimodal features such as color, motion and sound can be fused non-adaptively assuming conditional independence of the features given the state ${ }^{(15)}$. A consistent histogram for the analysis of color, edges, and texture features was proposed ${ }^{(16)}$. The features' noise parameters are changed adaptively and the features are adaptively weighted. Based on the local scale invariant feature transform (SIFT) methods were proposed ${ }^{(17)(18)}$. In the Ref. (17), the particle weight is calculated firstly by color similarity measurement and then updated according to the distribution of SIFT matches. However, only use the average values of the matches points of SIFT features to approximate the object center. In the Ref. (18), the Harris and SIFT feature are adopted to establish the object model, and Harris corner is applied to generate the SIFT feature vector which has significantly reduced the time complexity, and effectively tracked the object under the simple scene.

The paper is organized as follows: In Sect. 2, we explain the whole process and illustrate the flow chart of our proposed method. In Sect. 3, we introduce particle filter framework. The color feature and texture feature are presented in Sect. 4. In Sect. 5, we introduce extracting local feature points based SURF algorithm and propose an adaptive updating mechanism of local feature points. In Sect. 6, we present tracking window modification and particle weight updating. Finally, in Sects. 7 and 8, we summarize the experimental results and draw out conclusions.

\section{General Description}

As mentioned above, we propose a robust vehicle tracking method fused SURF feature, color feature and LBP texture. Numerious experiments and previous literatures show that a single feature is not fit to achieve target representation and discriminability. Such as, color feature has been widely used in the particle filter for likelihood estimation ${ }^{(3)-(5)}$, with characteristics like simple feature extraction and similarity calculation, insensitive to image scale and rotation. But it is prone to error when the background illumination changes or the target have little difference with background in color feature. Thus, the combinations of multiple features can be adopted to represent the target model and to improve the tracking performance. SURF method is a high-performance scale and rotation-invariant interest point detector and descriptor which approximates or even outperforms previously proposed schemes with respect to repeatability, distinctiveness, and robustness. Especially, the detection speed of feature point significantly faster than that of the similar algorithm, such as SIFT etc. This characteristic is convenient for engineering application. Otherwise, in this paper, after the local feature points of tracking target were extracted with SURF approach, in order to track quick appearance changes of target, and promptly adopt the new feature points reflecting the current situation of target, we propose the adaptive dynamic update mechanism which includes discarding bad feature points and adopting new feature points. Especially, the adopting mechanism of new feature points overcomes the shortcomings of randomly selected ${ }^{(6)}$ new points, enhances the adaptability of algorithm for tracking condition changes and improves the performance and reliability of tracking algorithm. Furthermore, we also propose the modification mechanism of tracking window, and the allocation mechanism of particle weights.

To facilitate understanding, we give the description and flow chart of our method as shown in Fig. 1. The whole process of our proposed tracking algorithm is described as follows:

Input: Video frames $F_{1}, F_{2}, \cdots, F_{n}$.

Output: Target regions according to target states in every frame.

Algorithm:

1). Select the target object in the initializing frame manually, and calculate the appearance model for the first frame.

2 ). Select and propagate samples at frame $F_{t}$ from the samples at $F_{t-1}$ according to their weights.

$3)$. Predict the state of particles according to the dynamic model.

4). Compute the weight of each feature using formula (6) and (12), and according to the likelihood using formula (9) and (15).

5). Extract SURF points and matching, compute the number of matching points.

$6)$. If the number of matching points is larger than a threshold, approximate the object center using formula (20) and (21), and update the weights of particles using the improved distance kernel function, else, only fuse the color and texture feature using formula (16).

7). Update SURF feature points of object target using formula (17) and (18).

8). Modify size of tracking window using formula (19).

9). Output target's location, and go to step 2.

\section{Particle Filter Framework}

Particle filter is an efficient statistical simulation method based on the idea of recursive Bayesian estimation. It uses a set of weighted particles sampled randomly to approximate the posterior possibility density function (pdf ${ }^{(19)}$. The state of a tracking object is described by the $x_{t}$ at time $t$ while $y_{t}$ denotes the observation of the system at time $t$. The key idea of particle filtering is to simulate the posterior distribution $p\left(x_{t} \mid y_{1: t}\right)$. In Bayesian theory, this can be formulated as iterative recursive process consisting of the prediction step $^{(20)}$

$$
p\left(x_{t} \mid y_{1: t-1}\right)=\int p\left(x_{t} \mid x_{t-1}\right) p\left(x_{t-1} \mid y_{1: t-1}\right) d x_{t-1} \cdots
$$

And of the update step

$$
p\left(x_{t} \mid y_{1: t}\right)=\frac{p\left(y_{t} \mid x_{t}\right) p\left(x_{t} \mid y_{1: t-1}\right)}{\int p\left(y_{t} \mid x_{t}\right) p\left(x_{t} \mid y_{1: t-1}\right) d x_{t}} \cdots \cdots \cdots \cdots
$$

The prediction step makes use of the available knowledge 


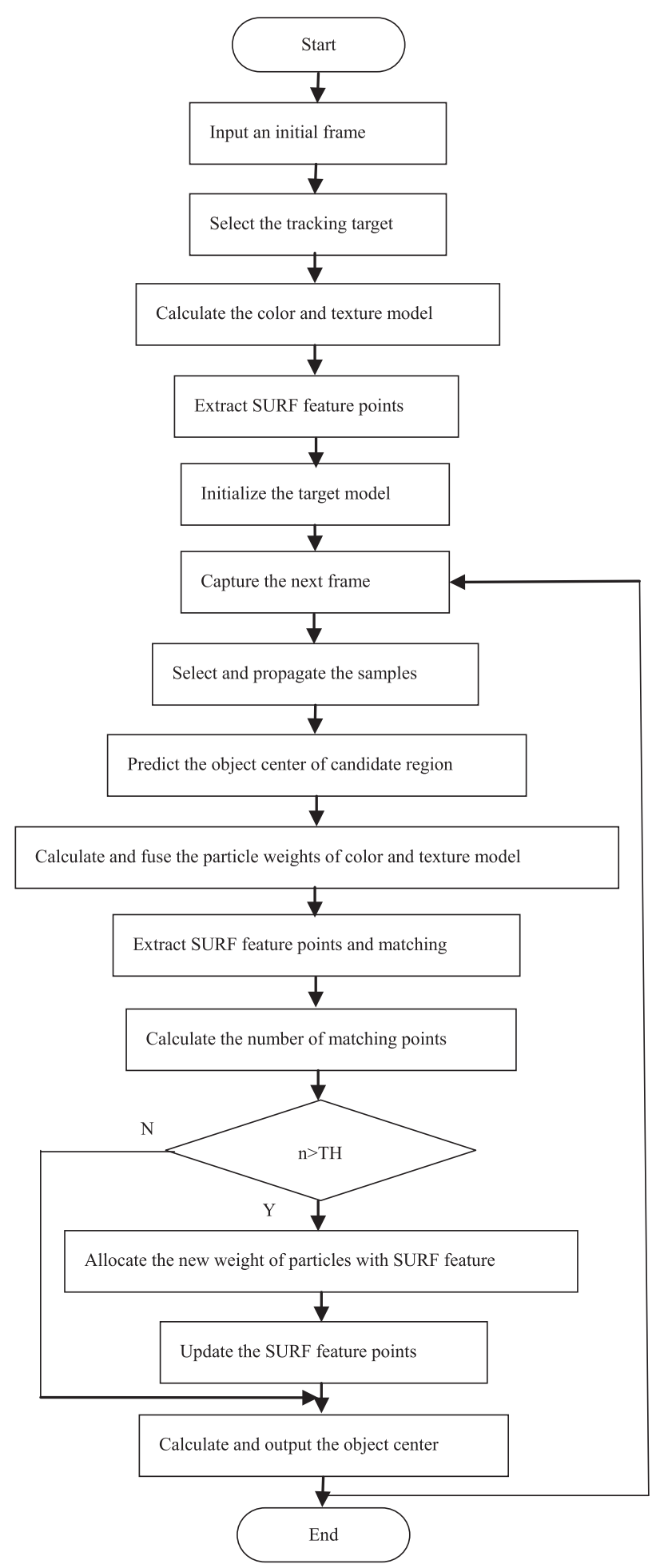

Fig. 1. The flow chart of our proposed method

of target evolution encoded in the dynamic model. The update step uses the measurement $y_{t}$ at time $t$, to update the predicted density. Where $p\left(x_{t} \mid x_{t-1}\right)$ is the dynamic model describing the state space evolution which corresponds to the evolution of the tracking object, and $p\left(y_{t} \mid x_{t}\right)$ is the likelihood of an observation $y_{t}$ given the state $\mathrm{x}_{\mathrm{t}}$. By applying the sequential importance sampling (SIS), the posterior distribution $p\left(x_{t} \mid y_{1: t}\right)$ can be approximated by a finite set of weighted samples $\left\{x_{t}^{(i)}, \omega_{t}^{(i)}\right\}_{i=1}^{N}$ as follows:

$$
p\left(x_{t} \mid y_{1: t}\right) \approx \sum_{i=1}^{N} \tilde{\omega}_{t}^{(i)} \delta\left(x_{t}-x_{t}^{(i)}\right) \ldots
$$

where $N$ is the number of samples, $\delta\left(x_{t}-x_{t}^{(i)}\right)$ is the Dirac delta function with mass at $x_{t}^{(i)}$. The variable $\tilde{\omega}_{t}^{(i)}$ is normalized so that $\tilde{\omega}_{t}^{(i)}=\frac{\omega_{t}^{(i)}}{\sum_{j=1}^{N} \omega_{t}^{(j)}}$, where particle weight $\omega_{t}^{(i)}$ is given as :

$$
\omega_{t}^{(i)}=\omega_{t-1}^{(i)} \frac{p\left(y_{t} \mid x_{t}^{(i)}\right) p\left(x_{t}^{(i)} \mid x_{t-1}^{(i)}\right)}{q\left(x_{t}^{(i)} \mid x_{0: t-1}^{(i)}, y_{1: t}\right)} \ldots \ldots \ldots \ldots \ldots \ldots
$$

The particles are sampled from a proposal distribution $x_{t}^{(i)} \sim q\left(x_{t}^{(i)} \mid x_{0: t-1}^{(i)}, y_{1: t}\right)$ (importance sampling). In our algorithm the proposal distribution is to use the prior density $q\left(x_{t}^{(i)} \mid x_{0: t-1}^{(i)}, y_{1: t}\right)=p\left(x_{t}^{(i)} \mid x_{t-1}^{(i)}\right)$. This kind of particle filter is known as condensation method or bootstrap filter ${ }^{(12)}$. To reduce the degeneracy problem, we resample the particle $\left\{x_{t}^{(i)}, \omega_{t}^{(i)}\right\}_{i=1}^{N}$ with replacement $N$ times according to the weights $\omega_{t}^{(i)}$. The resulting particles have uniformly distributed weights $\omega_{t}^{(i)}=1 / N$.

\section{Color Feature and Texture Feature}

4.1 Color Feature Color distributions are used as target models as they achieve robustness against non-rigidity, rotation and partial occlusion. In the RGB color space, because these three color components are reciprocally dependent, illumination changes have a great influence on the histogram, and the RGB space is not suffcient for discrimination under all circumstances, therefore, the HSV space is selected to represent color information. In the experiments, typical $8 \times 8 \times 4$ bins were used to make the histograms less sensitive to intensity variations. Suppose the distributions are discretized into $\mathrm{m}$ bins. The similar color distributions are also described by Comaniciu ${ }^{(21)}$. The histograms are produced with a function $b\left(x_{i}\right)$ that assigns the color of location $\mathrm{x}_{\mathrm{i}}$ to the corresponding bin. We calculate the color distribution in a rectangle region with long and short half axis $h_{x}, h_{y}$. To increase the reliability of the color distribution when boundary pixels belong to the background or get occluded, smaller weights are assigned to the pixels that are further away from the region center by employing a weighting function.

$$
k(\|r\|)=\left\{\begin{array}{c}
1-\|r\|^{2},\|r\|<1 \\
0, \quad \text { otherwise }
\end{array}\right.
$$

where $k(\cdot)$ is the Epanechnikov kernel, $r$ is the distance from the region center. Thus, the color distribution of target object $q_{c}=\left\{q_{c}^{(u)}\right\}_{u=1, \cdots, m}$ at position $x_{i}$ is defined as

$$
q_{c}^{(u)}=\mathrm{C} \sum_{i=1}^{n} k\left(\frac{\left\|x-x_{i}\right\|}{l}\right) \delta\left[b\left(x_{i}\right)-u\right]
$$

where $i$ is the number of pixels in the object region, $\delta$ is the Kronecker delta function, the parameter $l=\sqrt{h_{x}^{2}+h_{y}^{2}}$ is used to adapt the size of the region, and the normalization factor $\mathrm{C}$ ensures that $\sum_{u=1}^{m} q_{c}^{(u)}=1$.

In update step, we use similarity measures to calculate color distributions $p_{c}\left(x_{t}\right)=\left\{p_{c}^{(u)}\left(x_{t}\right)\right\}_{u=1, \cdots, m}$ of the candidate 
at time $t$. According to the color distributions of target object and candidate, the similarity measure between these two distributions is then given by the Hellinger distance ${ }^{(22)(23)}$.

$$
d_{c}\left[q_{c}, p_{c}\left(x_{t}\right)\right]=\sqrt{1-\rho\left[q_{c}, p_{c}\left(x_{t}\right)\right]} \ldots \ldots \ldots \ldots
$$

where $\rho\left[q_{c}, p_{c}\left(x_{t}\right)\right]$ is the Bhattacharyya coefficient that is defined as

$$
\rho\left[q_{c}, p_{c}\left(x_{t}\right)\right]=\sum_{u=1}^{m} \sqrt{q_{c}^{(u)} \cdot p_{c}^{(u)}\left(x_{t}\right)} \ldots \ldots \ldots \ldots \ldots
$$

The larger the measure $\rho$ is, the more similar these distributions. Conversely, for the distance $d_{c}$, the smaller the value is, the more similar these distributions. For two identical normalized histograms, if we obtain $d_{c}=0(\rho=1)$, indicating a perfect match.

The likelihood function for the color feature can be defined as

$$
p_{c}\left(y_{t} \mid x_{t}\right) \propto \exp \left(-\frac{d_{c}^{2}\left[q_{c}, p_{c}\left(x_{t}\right)\right]}{2 \sigma_{c}^{2}}\right) \ldots \ldots \ldots \ldots \ldots
$$

where, the standard deviation $\sigma_{c}$ specifies the Gaussian noise in the measurements. Note that small Hellinger distances correspond to large weights in the particle filter. Calculate the each particle's similarity and normalize to gain the weight $w_{t}^{C}$.

4.2 Texture Feature Texture is an important characteristic of describing the target, but only a limited number of examples of successful exploitation of texture exist. A major problem is that textures in the real world are often not uniform due to variations in orientation, scale, or other visual appearance ${ }^{(24)}$. In addition, the degree of computational complexity of most proposed texture measures is too high. These measures are unable to meet the real-time tracking needs. In this paper, we adopt the LBP texture operator ${ }^{(25)}$, which has recently shown excellent performance in many applications. LBP is a gray-scale invariant texture primitive statistic. The operator labels the pixels of an image region by thresholding the neighborhood of each pixel with the center value and considering the result as a binary pattern. The LBP texture operator can be defined as

$$
\operatorname{LBP}_{P, R}\left(x_{c}, y_{c}\right)=\sum_{p=0}^{P-1} s\left(g_{p}-g_{c}\right) 2^{p} \ldots \ldots \ldots \ldots \ldots
$$

where $g_{c}$ corresponds to the gray value of the center pixel $\left(x_{c}, y_{c}\right)$ of a local neighborhood and $g_{p}$ to the gray values of $P$ equally spaced pixels on a circle of radius $R$. The function $s(x)$ is defined as

$$
s(x)= \begin{cases}1, & x \geq \operatorname{th} 1 \\ 0, & x<\operatorname{th} 1\end{cases}
$$

where $t h 1$ is a given threshold which makes the LBP more robust against these negligible changes in pixel values. In our experiments, we set $P=6, R=3$ and $t h 1=3$.

Thus, the texture histogram of the image $I\left(x_{i}, y_{i}\right)$ can be defined as

$$
q_{L}^{(u)}=\sum_{i=1}^{n} F\left\{L B P\left(x_{i}, y_{i}\right)=u\right\}
$$

where $u=0, \cdots, m-1$; As described above. The LBP operator is separated into $m$ bins. In our experiment, $m$ is equal to 64. The function $F\{\cdot\}$ is defined as

$$
F\{A\}= \begin{cases}1, & A \text { is True } \\ 0, & A \text { is False }\end{cases}
$$

Suppose the texture distribution of target object is $q_{L}=$ $\left\{q_{L}^{(u)}\right\}_{u=1, \cdots, m}$, the texture distribution of candidate is $p_{L}\left(x_{t}\right)=$ $\left\{p_{L}^{(u)}\left(x_{t}\right)\right\}_{u=1, \cdots, m}$ at time $t$.

The similarity measure between these two distributions is described as

$$
d_{L}\left[q_{L}, p_{L}\left(x_{t}\right)\right]=1-\sum_{u=1}^{m} \min \left[q_{L}^{(u)}, p_{L}^{(u)}\left(x_{t}\right)\right] \cdots \cdots
$$

This measure has an intuitive motivation in that it calculates the common part of two histograms. It explicitly neglects features which only occur in one of the histograms ${ }^{(25)}$.

Thus, the likelihood function for the texture feature can be defined as

$$
p_{L}\left(y_{t} \mid x_{t}\right) \propto \exp \left(-\frac{d_{L}^{2}\left[q_{L}, p_{L}\left(x_{t}\right)\right]}{2 \sigma_{L}^{2}}\right) \ldots \ldots \ldots \ldots \ldots
$$

Here, the standard deviation $\sigma_{L}$ specifies the Gaussian noise in the measurements. Thus, we calculate the each particle's similarity measure and normalize to gain the weight $w_{t}^{L}$. Multiple feature fusion can better utilize information provided by the features supplementing each other and improve the robustness of the algorithm. The relationship between different features has been treated differently by different authors. In this paper, we assume that the relationships among color, texture and SURF feature are independent. Thus we can obtain the fusion weighting by:

$$
w_{t}^{F}=\gamma w_{t}^{C}+(1-\gamma) w_{t}^{L}
$$

where $\gamma \in[0,1]$ is an adaptive control parameter. And $\gamma$ denotes the proportion of the number of larger weight color particle to the total number of color and texture particles. Note that the larger the value, the more similar color feature distributions.

\section{SURF Feature}

The SURF algorithm is a novel scale and rotation invariant interest point detector and descriptor. It approximates or even outperforms previously proposed schemes with respect to repeatability, distinctiveness, and robustness, and yet it can be computed and compared much faster ${ }^{(26)(27)}$. In interest point detection, it uses a very basic Hessian matrix approximation with box filters. These approximate second order Gaussian derivatives and can be evaluated at a very low computational cost using integral images. Also, about scale space representation, the SURF algorithm apply box filters of any size at exactly the same speed directly on the original image and even in parallel to achieve a higher level of the image pyramid. In interest point description, it is built on the distribution of first order Haar wavelet responses in $\mathrm{x}$ and $\mathrm{y}$ direction rather than the gradient, exploit integral images for speed, and use only 64D. This reduces the time for feature computation, and has proven to simultaneously increase the robustness of the 


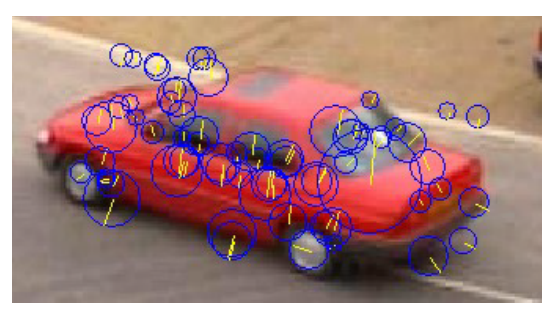

Fig. 2. Detail of the vehicle showing the size of the oriented descriptor window at different scales

\section{descriptor}

The SURF feature descriptor is represented as $S=$ $\{x, y, s, o, h i s t\}$, where $x$ and $y$ are the position of the feature in terms of the image coordinates, $s$ is the feature scale, $o$ is the feature vector direction, and hist is the gradient orientation distribution quantized into 64 or 128 bins.

5.1 SURF Feature Point Matching In the matching strategy, several matching methods are defined, such as fast indexing matching, nearest neighbor matching $(\mathrm{NN})$, and nearest neighbor distance ratio (NNDR). In this paper, we use the NNDR approach ${ }^{(28)}$. This approach is similar to nearest neighbor matching, except that the thresholding is applied to the distance ratio between the first and the second nearest neighbor. For two regions $\mathrm{A}$ and $\mathrm{B}$, the regions are matched if $\left\|D_{A}-D_{B}\right\| /\left\|D_{A}-D_{C}\right\|<t$, where $D_{B}$ is the first and $D_{C}$ is the second nearest neighbor to $D_{A}, \mathrm{t}$ is experimental threshold and is set to be 0.5 in our work. Because the precision of each SURF feature matching point may affect the tracking in our experiment, especially when the mismatched feature points are far away from the object center. So, before matching, we firstly discard the outliers of feature points using the random sample consensus (RANSAC) algorithm ${ }^{\text {(29)(30). }}$.

5.2 Features Points Update Mechanism In the tracking stage, due to the deformation of moving target, we must update the feature points of the target template over time. Therefore we propose the adaptive on-line update mechanism. It includes two stages, discarding bad feature points and adopting new feature points.

5.2.1 Discarding Bad Feature Points Our hypothesis is that the repeated occurrences of a feature point within the target template in the past period of time is rarely, then the probability of re-emergence of this feature point in the next period of time is almost zero. Based on this assumption, we examine the repeated occurrences of every point in target template for a while, to measure whether we want to discard or adopt this point. This similar method was introduced in Refs. (6), (31). The mechanism is defined as

$$
P_{i, t+1}=(1-\theta) \cdot P_{i, t}+\theta \cdot \delta
$$

where $P_{i, t+1}$ is the probability of the ith feature point being in the target template at time $t+1$ and $\theta \in[0,1]$ indicates the degree of object change. The larger $\theta$ is, the faster the object will change, and $\delta_{i} \in\{0,1\}$ is a factor of matching determination procedure. If the current ith feature point in target template match any one of candidate feature points, $\delta_{i}=1$, else $\delta_{i}=0$. If the probability $P_{i, t+1}$ is greater than an experimental threshold $t h 2$, show that the feature point is a stable feature point, in other words, the frequency of its occurrence in consecutive frames is large. Otherwise, we will discard

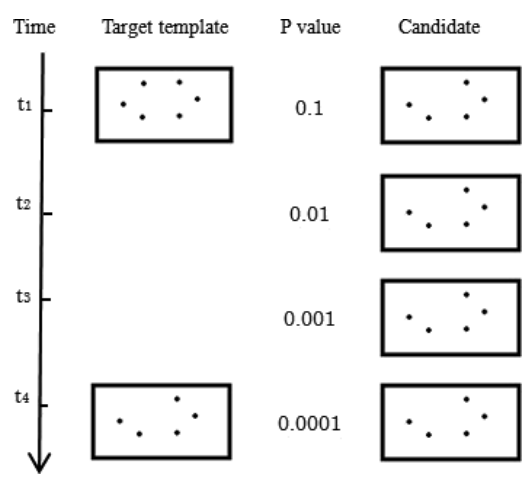

Fig. 3. Discarding the upper left point of the target template at time $t_{4}$

this point from the template. In our work, th 2 is set to be 0.0001. As shown in Fig. 3, at time $t_{4}$, we will discard the upper left point of the target template according to this mechanism. The benefit of this mechanism is that it can quickly adapt to the appearance changes of the tracking object, and instantly delete the inappropriate feature points.

5.2.2 Adopting New Feature Points In order to adapt to the quick appearance changes of target, the tracker should not only delete inappropriate old features points, but also promptly adopt new feature points reflecting the current situation of target. In the existing algorithms which were presented in Refs. (6), (31), with the advent of new frame, the feature points randomly selected from the new frame are added to the target template. The random selection method brings uncertainty to the object template and is not conducive to the stability of the tracking. Therefore, we present our hypothesis. The adopted new feature points should be selected from a stable non-matching set of the candidate objects with the target template for a while.

In our experiment, we need to define the object template. Given a feature point of object template set $S_{t_{0}}=$ $\left\{s_{1}, s_{2}, \cdots, s_{n}\right\}$, as mentioned above, the weight corresponding to each feature point is $P_{i, t}$ at time $t$. Now assuming the target object has been successfully tracked in frame $I_{t-1}$. When frame $I_{t}$ arrives, we detect the candidate feature point set $C_{t}=\left\{c_{1}, c_{2}, \cdots, c_{q}\right\}$ using the SURF detector and descriptor and get the matching candidate set $M_{t}=\left\{m_{1}, m_{2}, \cdots, m_{p}\right\}$ and un-matching set $U_{t}=\left\{u_{1}, u_{2}, \cdots, u_{l}\right\}$ with the template set $S_{t}$ by the NNDR method, where $M_{t} \cup U_{t}=C_{t}$, $M_{t} \cap U_{t}=\emptyset, p+l=q . s_{i} c_{i}, m_{i}$ and $u_{i}$ denote the state of the ith feature point from the corresponding feature points sets $S_{t}, C_{t}, M_{t}$ and $U_{t}$ at time $t$ respectively. Next, when new frame $I_{t+1}$ arrives, we can get the new candidate feature point set $C_{t+1}$ according to the prediction step of particle filter, and, after matching the object template $S_{t+1}$, also get the matching candidate set $M_{t+1}$ and un-matching set $U_{t+1}$. For the two consecutive un-matching sets $U_{t}$ and $U_{t+1}$, in order to detect the stability of each point of these two sets, we define a new matching set $U M_{t+1}$ which contains the matching points of the two consecutive un-matching sets $U_{t}$ and $U_{t+1}$. If the two sets do not match, we use the set $U_{t+1}$ to represent the set $U M_{t+1}$, and compute the each point probability by

$$
U P_{i, t+1}=\left\{\begin{aligned}
U P_{i, t}+1, & \text { if matching is true } \\
1, & \text { otherwise }
\end{aligned}\right.
$$




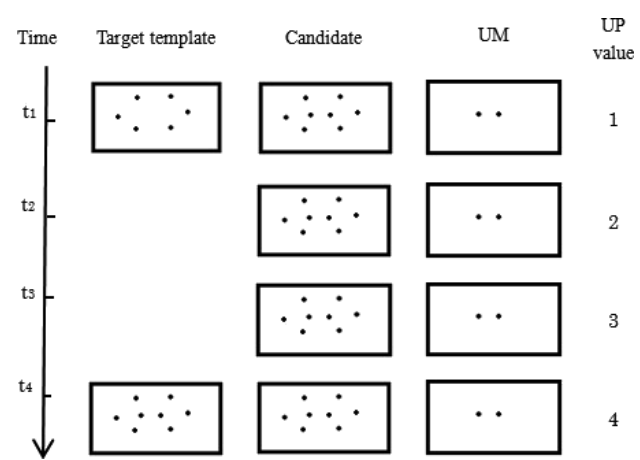

Fig. 4. Adopting the two stable center points from the non-matching set UM at time $t_{4}$

where $U P_{i, t}$ represents the weight of the point in the matching set $U M_{t}$ that matches the ith point in the un-matching set $U_{t+1}$. If the feature probability $U P_{i, t+1}$ exceeds a threshold $t h 3$, it represents that this point is a stable non-matching point. Thus we will adopt this point to the object template. In this experiment, th 3 is set to be 4 , if the feature point exists in more than three consecutive frames, we will adopt this point. As shown in Fig. 4, we will adopt the two stable center points from the non-matching set UM at time $t_{4}$ according to this mechanism. This mechanism of adopting new feature point overcomes the shortcomings of randomly selected new points. And experiments show that has a strong adaptability for tracking condition changes.

\section{Tracking Algorithm of Fusion Multiple Fea- ture}

6.1 Tracking Window Modification In our paper, the tracking window is described as a rectangle region $h=$ $\left\{x, y, h_{x}, h_{y}\right\}$, where $x$ and $y$ denote the center coordinates of the rectangle region, $h_{x}$ and $h_{y}$ represent the long and short half axis. In the practical tracking, the object may change its size with motion of object itself, and distance between the object and camera. To keep precise tracking, the size of tracking window should be modified automatically with the state of the object. Considering the size of color region, texture region and SURF matches distribution region in current frame, and the tracking window size in the former frame, we can obtain the location $x$ and $y$, and size $h_{x}$ and $h_{y}$ of tracking windows in current frame at time $t$ by

$$
\begin{aligned}
h_{t}= & \lambda h_{t-1}+(1-\lambda)\left((\alpha+\beta) h_{S U R F}+(1-\alpha) h_{\text {color }}\right. \\
& \left.+(1-\beta) h_{\text {texture }}\right) \ldots \ldots \ldots \ldots \ldots \ldots \ldots \ldots
\end{aligned}
$$

where $h_{\text {SURF }}$ represents the window covering the region of SURF matches distribution, $h_{\text {color }}$ and $h_{\text {texture }}$ denote the window region that cover the color and texture region of object, respectively. $\alpha, \beta$ is a harmonizing factor that balances the effect of SURF cue, color cue and texture cue, $\lambda$ is a forgetting factor that balances the window in frames at time $t$ and time $t-1$ to avoid over-sensitive scale adaption. The similar approach is proposed in Refs. (17), (32).

6.2 Particle Weight Updating In the tracking process, there are complex conditions such as illumination change, partial occlusion, and similar background. Single feature is unable to meet tracking needs, although the computational complexity of weighted algorithm is low. In our paper, we combine the color feature, texture feature and the SURF feature distributions, and present an improved distance kernel function to update the weights of particles to achieve adaptive tracking.

Suppose the state of particles at time $t$ is defined as $X_{t}=$ $\left\{x_{i}, \dot{x}_{i}, y_{i}, \dot{y}_{i}, h_{x i}, h_{y i}\right\} i=1, \cdots, N$, where $N$ represents the total number of particles, $x$ and $y$ represent the center coordinates of the rectangular box for tracking an object, $\dot{x}$ and $\dot{y}$ represent the respective velocity components, $h_{x}$ and $h_{y}$ denote long and short half axis of rectangle region, respectively. $w_{t}=\left\{w_{i}^{(t)}\right\}$ represents the weight in which $w_{t}$ is computed by $w_{t}^{F}$. The SURF feature matching candidate set is $M_{t}=\left\{m_{1}, m_{2}, \cdots, m_{p}\right\}$.

Thus, the weight of each particle is allocated as follows:

Step 1. Approximate the object center $\mathrm{C}(\mathrm{x}, \mathrm{y})$ by

$$
\begin{aligned}
& x=\varepsilon\left(\sum_{i=1}^{N} x_{i} w_{i}^{F}\right)+(1-\varepsilon)\left(\frac{1}{P} \sum_{i=1}^{P} x_{i}^{S}\right) \ldots \ldots \ldots \\
& y=\varepsilon\left(\sum_{i=1}^{N} y_{i} w_{i}^{F}\right)+(1-\varepsilon)\left(\frac{1}{P} \sum_{i=1}^{P} y_{i}^{S}\right) \ldots \ldots \ldots \ldots
\end{aligned}
$$

Step 2. For $i=1, \cdots, N$

(a) Calculate the Euclidean distance $d_{i}$ from each particle to center point $\mathrm{C}$.

(b) Get the factors using exponential kernel function.

$$
k_{S}\left(d_{i}\right)=\left\{\begin{array}{cl}
1, & d_{i} \leq T_{d} \\
\exp \left((-1)\left(d_{i}-\frac{T_{d}}{\max \left\{d_{i}-T_{d}\right\}_{i=1}^{N}}\right)\right), & d_{i} \geq T_{d}
\end{array}\right.
$$

(c) Update the weight $w_{i}$ using

$$
w_{i}^{(t)}=k_{S}\left(d_{i}\right) \cdot w_{i}^{(t)} \ldots
$$

Step 3. Normalize the weights

$$
\tilde{w}_{i}^{(t)}=w_{i}^{(t)} / \sum_{i=1}^{N} w_{i}^{(t)}
$$

where, $x_{i}^{S}$ and $y_{i}^{S}$ are the coordinates of SURF feature points, $\varepsilon$ is a weight of fusion three feature and is set to be 0.4 . $T_{d}$ is set as the half of the minimum of width and height of object rectangular region.

\section{Experiments Results and Analysis}

7.1 Test Conditions In order to verify the effectiveness of proposed algorithms, we demonstrate the proposed tracker on a data set from three different tracking sequences (Table 1). The vehicles (V1 and V2) are extracted from the PETS2001 ${ }^{(34)}$ and PETS2000 ${ }^{(33)}$ data set, respectively. Finally, the vehicle (V3) is extracted from a traffic surveillance video. Because of the different characteristics of target motion, such as, significant deformation and target rotation under curvilinear motion, these characteristics bring larger challenges to track tasks.

The parameters of the tracker are set experimentally, the forgetting factor $\lambda$ and $\theta$ are set to be 0.9. $\alpha$ and $\beta$ are set to be 0.5 . Note that the SURF based weight updating could be applied only when there are many enough SURF matching points, we set the least threshold as 5. The 200 particles are 
Table 1. The description of the tracking data set

\begin{tabular}{l|l|l|l|l}
\hline Targets & Frame size & Frame rate & $\begin{array}{l}\text { Target } \\
\text { trajectory }\end{array}$ & Characteristics \\
\hline V1 & $768 * 576$ & $25 \mathrm{fps}$ & slash & $\begin{array}{l}\text { Scale changes, } \\
\text { clutter, fast motion }\end{array}$ \\
\hline V2 & $768 * 576$ & $25 \mathrm{fps}$ & curve & $\begin{array}{l}\text { Scale changes, } \\
\text { rotation }\end{array}$ \\
\hline V3 & $800 * 600$ & $30 \mathrm{fps}$ & $\begin{array}{l}\text { Straight } \\
\text { line }\end{array}$ & $\begin{array}{l}\text { Scale changes, } \\
\text { illumination changes }\end{array}$ \\
\hline
\end{tabular}

used for per frame. The results presented were obtained from MATLAB implementation with a Core $3.40 \mathrm{GHz}$ PC under Windows.

7.2 Performance Evaluation and Comparison In order to highlight the difference of tracking results with different features and different tracking algorithms, we implement some state-of-the art trackers including the combining color and the SIFT feature particle filter-based tracker ${ }^{(17)}$ (referred to as SIFT-PF), the standard mean shift tracker $^{(7)}$ and the single color feature tracker. The SIFT-PF is a multiple feature tracker, but, which only uses the color histogram for object representation, uses SIFT points to approximate the object center. The mean-shift tracker based on histogram analysis is a classical tracking algorithm. It is a mode-finding technique that locates the local minimum of the posterior density function. Note that, in addition to the Mean shift method, the other trackers (including ours) utilize the same particle filtering framework for tracking and use the same dynamic model in the experiments. The implementations are all parameterized according to the original paper with some tuning. Therefore, the comparison is valid because only the observation model changes between our tracker and the other two trackers. And everything else is kept consistent. We present some representative frames to show the tracking results and also give some statistical analysis to quantify the performance of the proposed tracker.

The first evaluation criteria of the tracking error are based on the relative position errors (in pixel) between the center of the tracking result and that of the ground truth. Ideally, the position differences should be around zero.

The first sequence undergoes a significant scale changes, fast motion and clutter environment. Some samples of the final tracking results are demonstrated in Fig. 5, where rows 1, 2, 3 and 4 correspond to mean shift, Color-PF, SIFT-PF and our tracker, respectively, in which three representative frames $(313,352,375)$ are shown. It shows a blue color vehicle moving from the left top corner of the image to the right bottom. Note that the vehicle is small in the image and undergoes scale changes and fast motion. The number of SURF feature point is smaller, thus the performances of our proposed tracker is slightly better than the other three trackers. Since the background undergoes clutter environment, the position error of these three methods is larger between 340th and 360th frame, but all three methods can locate the vehicle position. The quantitative comparison of the tracker in terms of position errors is shown in Fig. 8.

For second sequence V2, the tracking results are illustrated in Fig. 6, where rows 1, 2, 3 and 4 correspond to mean shift, Color-PF, SIFT-PF and our tracker, respectively, in which

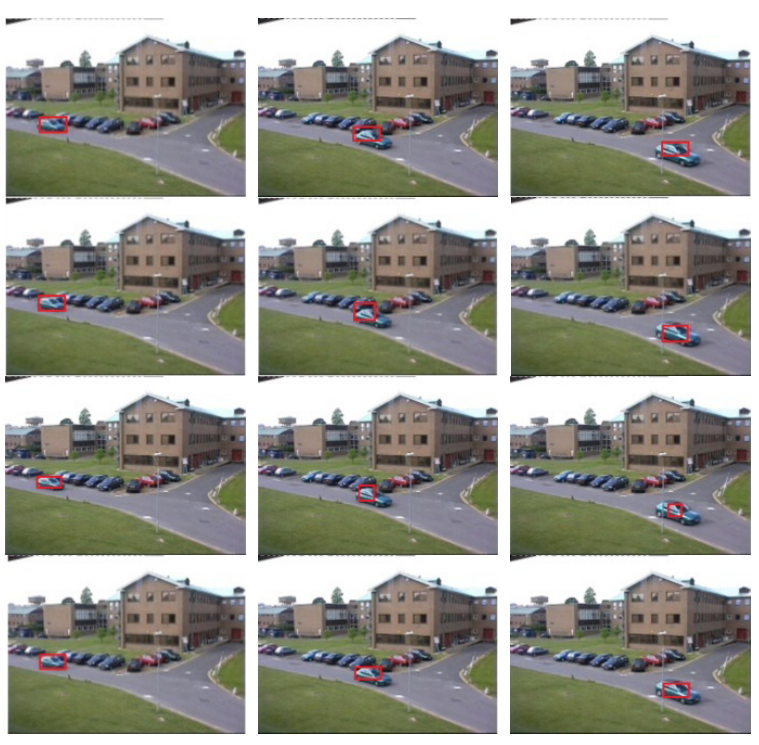

Fig. 5. Tracking results of sequence V1 as it undergoes scale changes and clutter. Rows 1, 2, 3 and 4 correspond to Mean shift, Color-PF, SIFT-PF, and our tracker, respectively
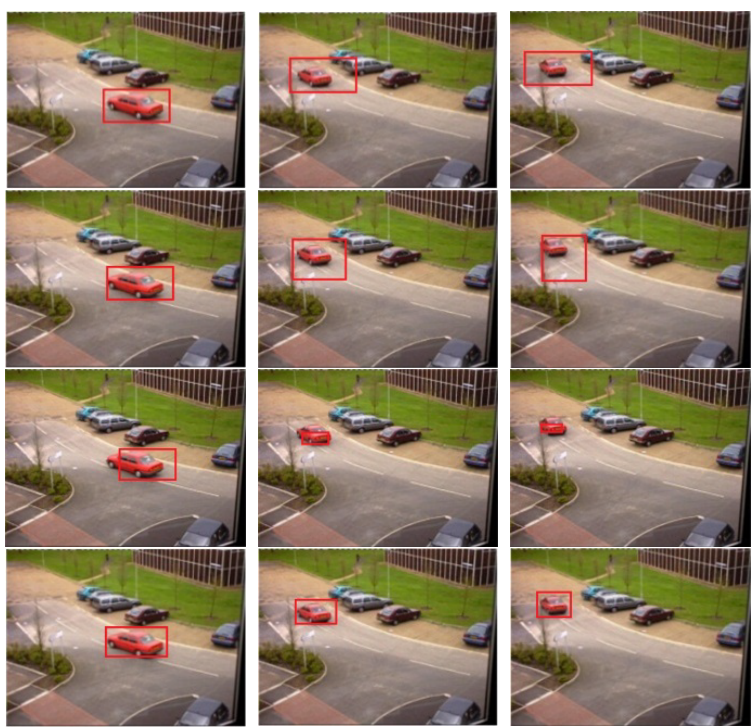

Fig. 6. Tracking results of sequence V2 as it undergoes scale changes and rotation. Rows $1,2,3$ and 4 correspond to Mean shift, Color-PF, SIFT-PF and our tracker, respectively

three representative frames $(140,175,189)$ are shown. The characteristics of target motion are significant deformation and target rotation under curvilinear motion. The rotation invariant detectors and descriptors of SURF algorithm offer a good compromise between feature complexity and robustness to commonly occurring photometric deformations. The tracking results show the mean shift tracker (rows 1) and PF-color tracker (rows 2) have large position errors in this challenging situation. And the PF-color tracker even occur tracking failure. While SIFT-PF drifts gradually, because it only approximates the center point of tracking object using the SIFT feature points. The evaluation results of these four algorithms are shown in Fig. 9.

The last sequence V3 is a traffic surveillance video which undergoes scale changes and illumination changes. Some 


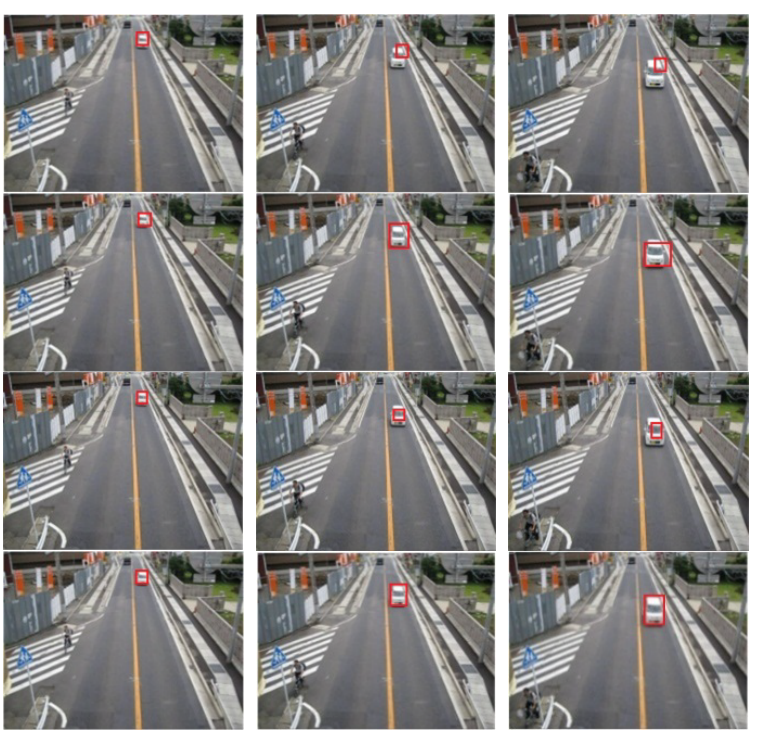

Fig. 7. Tracking results of sequence $\mathrm{V} 3$ as it undergoes scale changes and illumination changes. Rows 1, 2, 3 and 4 correspond to mean shift, Color-PF, SIFT-PF, and our tracker, respectively

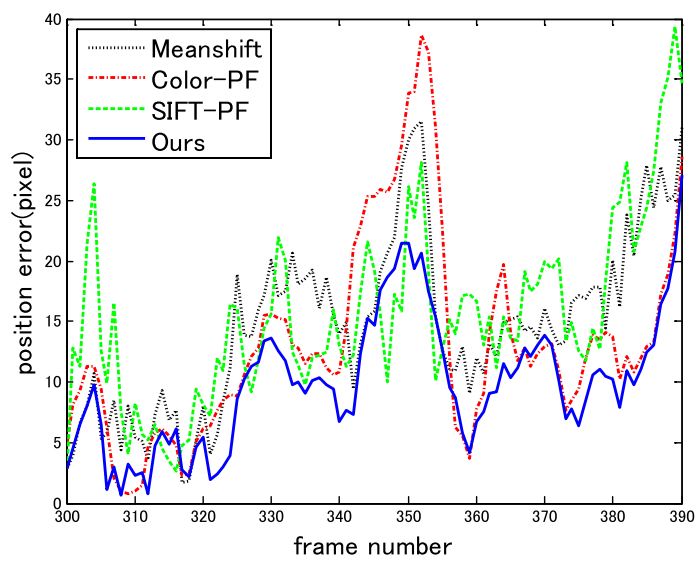

Fig. 8. Evaluation results of V1

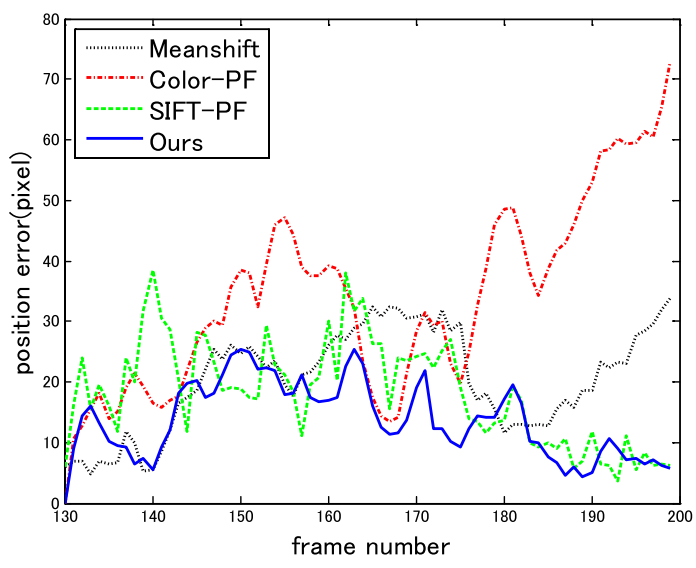

Fig. 9. Evaluation results of V2

samples of the tracking results are shown in Fig. 7, where rows 1, 2, 3 and 4 correspond to mean shift, Color-PF, SIFT$\mathrm{PF}$ and our tracker, respectively, in which three representative frames $(50,85,103)$ are shown. The significant deformation of the moving vehicle leads to poor performance of the mean shift tracker (rows 1). Moreover, the PF-color tracker

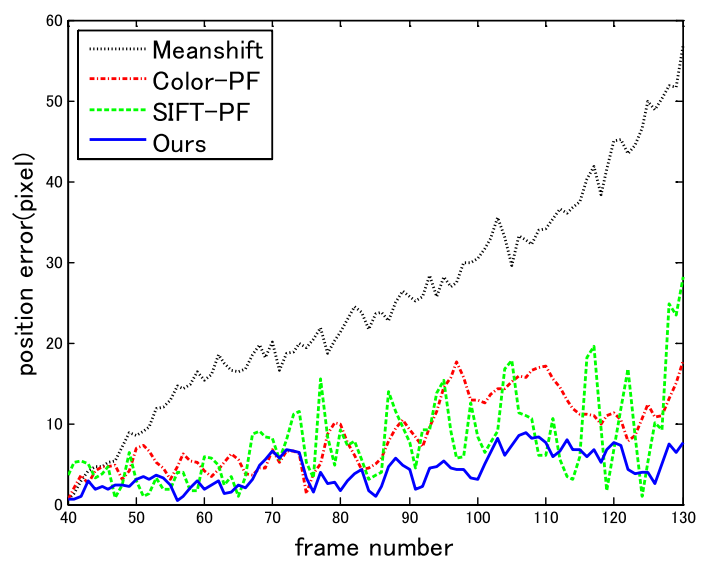

Fig. 10. Evaluation results of V3

Table 2. P-value evaluation of the tracking data set

\begin{tabular}{l|l|l|l|l}
\hline Target & Mean shift & Color-PF & SIFT-PF & Proposed method \\
\hline V1 & 0.378 & 0.433 & 0.556 & 0.352 \\
\hline V2 & 0.404 & 0.426 & 0.351 & 0.193 \\
\hline V3 & 0.565 & 0.280 & 0.503 & 0.194 \\
\hline
\end{tabular}

cannot provide accurate state information for the moving vehicle. Note that the vehicle is becoming larger, more and more the SURF feature points are captured, the performances of out proposed method are significantly improved. SIFT-PF also succeeds in tracking the target but has a larger tracking error compared to our method, and we can see obviously that SIFT-PF changes the position of target drastically because of the approximate method of the center point of object. SIFT-PF only uses the SIFT feature points. On the contrary, when the vehicle starts pulling away, it becomes smaller and smaller since the number of feature point is small, the performances of our method are similar to the classical particle filter. Fig. 10 shows the evaluation results of comparison.

The second evaluation method was proposed in Ref. (35), the error measure quantifies the discrepancy between the target estimations and the manually generated ground truth targets. This evaluation method was this performance measure rewards candidates with a high percentage of true positive pixels, and with few false positives and false negatives avoiding the asymmetry problem of other area based measures. Let $\mathrm{T}_{\mathrm{p}}$ be the number of true positive pixels (i.e., pixels belonging to both ground truth target and estimated target) in each current frame. Let $|\cdot|$ denote the cardinality of set. If $\left|A_{g}\right|$ and $\left|A_{e}\right|$ are the ground-truth and the estimated target area respectively, then the error of the estimation $\mathrm{P}$ in current frame can be defined as

$$
\mathrm{P}=1-\frac{2 \mathrm{~T}_{\mathrm{p}}}{\left|\mathrm{A}_{\mathrm{e}}\right|+\left|\mathrm{A}_{\mathrm{g}}\right|}
$$

In our experiment, the quality measure of a whole track is obtained by averaging $\mathrm{P}$ value over the frames where the target is visible. Since particle filter is a probabilistic algorithm, each tracker is run 50 times for each sequence. The comparison results of these four algorithms are shown in Table 2. We can see obviously that the target estimation of our tracker is more precise than other trackers.

For the efficiency of algorithm, the computational complexity of proposed method is mainly focused on the 
extracting and matching of local feature points. Under the non-optimal condition, comparing the efficiency of the proposed method and SIFT-PF, our approach of applying the incremental SURF detection takes $128.5 \mathrm{~ms}$ on average, the latter takes $168.3 \mathrm{~ms}$. Obviously, our algorithm is superior to contrast method about the computational cost, but, the improving of efficiency will be still considered in our future work.

\section{Conclusion}

In this paper, we propose a multiple features matching method based on SURF feature for robust vehicle tracking, For further robustness, we propose an adaptive update mechanism of feature point including discarding bad feature points and adopting the new feature points, and improve the distance kernel function method to allocate the weight of particle. In thorough experiments involving challenging sequences and other state-of-the-art trackers, our approach demonstrates very promising performance. The experimental results clearly demonstrate the effectiveness of our propose method.

\section{Acknowledgment}

This work was supported by the national natural science foundation of China (Grant No.61272283) and the specialized research program of the educational office of Shaanxi province (No.09JK645).

\section{References}

( 1 ) W. Hu, T. Tan, L. Wang, and S. Maybank: "A survey on visual surveillance of object motion and behaviors", IEEE Trans. on Systems, Man, and Cybernetics, Part C: Applications and Reviews, Vol.34, No.3, pp.334-352 (2004)

( 2 ) J. Scharcanski, A.B. deOliveira, P.G. Cavalcanti, and Y. Yari: "A particlefiltering approach for vehicular tracking adaptive to occlusions", IEEE Trans. on Vehicular Technology, Vol.60, No.2, pp.381-389 (2011)

( 3 ) K. Numiaro, E. Koller-Meier, and L.V. Gool: "An adaptive color-based particle filter", Image and Vision Computing, Vol.21, No.1, pp.99-110 (2003)

( 4 ) K. Numiaro, E. Koller-Meier, and L.V. Gool: "Object tracking with an adaptive color-based particle filter", Pattern Recognition 24th DAGM Symposium 2002, Lect. Notes Comput. Sci., Vol.2449, pp.353-360 (2002)

( 5 ) Y. Rui and Y. Chen: "Better proposal distribution object tracking using unscented particle filter", IEEE Computer Society Conf. on Computer Vision and Pattern Recognition, Vol.2, pp.786-793 (2001)

( 6 ) Q. Miao, G. Wang, C. Shi, X. Lin, and Z. Ruan: "A new framework for online object tracking based on SURF", Pattern Recognition Letters, Vol.32, pp.1564-1571 (2011)

( 7 ) D. Comaniciu, V. Ramesh, and P. Meer: "Kernel-based object tracking", IEEE Trans. on Pattern Analysis and Machine Intelligence, Vol.25, No.5, pp.564-567 (2003)

( 8 ) S. Spor and R. Rabenstein: "A real-time face tracker for color video", IEEE Int. Conf. on Acoustics, Speech and Signal Processing, Vol.3, pp.1493-1496 (2001)

( 9 ) D. Koller, J. Weber, and J. Malik: "Towards real time visual based tracking in cluttered traffic scenes", Proc. of the Intelligent Vehicles'94 Symposium, pp.201-206 (1994)

(10) Mikic, M. Trivedi, E. Hunter, and P. Cosman: "Human body model acquisition and tracking using voxel data", International Journal of Computer Vision, Vol.53, No.3, pp.199-223 (2003)

(11) J.J. LaViola: "A comparison of unscented and extended Kalman filtering for estimating quaternion motion", Proc. of American Control Conference, Vol.3, pp.2435-2440 (2003)

(12) A. Doucet, N.D. Freitas, and N. Gordon: "Sequential Monte Carlo methods in practice", Springer-Verlag, New York (2001)

(13) E. Maggio and A. Cavallaro: "Multi-part target representation for color tracking", Proc. IEEE Int. Conf. on Image Processing, Vol.2, pp.729-731 (2005)

(14) C. Shen, A.V.D. Hengel, and A. Dick: "Probabilistic multiple feature integration for particle filter based tracking", Proc. 7th Int. Conf. Digital Image
Computing, pp.309-408 (2003)

(15) P. Perez, J. Vermaak, and A. Blake: "Data fusion for visual tracking with particles", Proc. IEEE, Vol.92, No.3, pp.495-513 (2004)

(16) P. Brasnett, L. Mihaylova, D. Bull, and N. Canagarajah: "Sequential Monte Carlo tracking by fusing multiple features in video sequences", Image and Vision Computing, Vol.25, No.8, pp.1217-1227 (2007)

(17) P. Wu, L. Kong, F. Zhao, and X. Li: "Particle filter tracking based on color and SIFT features", Int. Conf. on Audio, Language and Image Processing, pp.932-937 (2008)

(18) Q. Zhang, T. Rui, H. Fang, and J. Zhang: "Particle filter object tracking based on Harris-SIFT feature matching", Procedia Engineering, Vol.29, pp.924929 (2012)

(19) N.J. Gordon, D.J. Salmond, and A.F.M. Smith: "Novel approach to nonlinear/non-Gaussian Bayesian state estimation", IEEE Proceedings of Radar and Signal Processing, Vol.140, No.2, pp.107-113 (1993)

(20) M.S. Arulampalam, S. Maskell, N. Gordon, and T. Clapp: "A tutorial on particle filters for online nonlinear/non-Gaussian Bayesian tracking", IEEE Trans. on Signal Processing, Vol. 50, No.2, pp.174-188 (2002)

(21) D. Comaniciu, V. Ramesh, and P. Meer: "Real-time tracking of non-rigid objects using mean shift", Proc. IEEE Int. Conf. on Computer Vision and Pattern Recognition, Vol.2, pp.142-149 (2000)

(22) F. Aherne, N. Thacker, and P. Rockett: "The Bhattacharyya metric as an absolute similarity measure for frequency coded data”, Kybernetika, Vol.34, No.4, pp.363-368 (1998)

(23) T. Kailath: "The divergence and Bhattacharyya distance measures in signal selection", IEEE Trans. on Communication Technology, Vol.15, No.1, pp.5260 (1967)

(24) T. Ojala, M. Pietikainen, and T. Maenpaa: "Multi-resolution grayscale and rotation invariant texture classification with local binary patterns", IEEE Trans. on Pattern Analysis and Machine Intelligence, Vol.24, No.7, pp.971987 (2002)

(25) M. Heikkila and M. Pietikainen: "A texture-based method for modeling the background and detecting moving objects", IEEE Trans. on Pattern Analysis and Machine Intelligence, Vol.28, No.4, pp.657-662 (2006)

(26) H. Bay, T. Tuytelaars, and L.Van Gool: "SURF: speeded up robust features", Computer Vision-ECCV 2006, Lecture Notes in Computer Science, Vol.3951, pp.404-417 (2006)

(27) H. Bay, A. Ess, T. Tuytelaars, and L. Van Gool: "Speeded-Up Robust Features (SURF)", Computer Vision and Image Understanding, Vol.110, pp.346-359 (2008)

(28) K. Mikolajczyk and C. Schmid: "A performance evaluation of local descriptors", IEEE Trans. on Pattern Analysis and Machine Intelligence, Vol.27, No.10, pp.1615-1630 (2005)

(29) M.A. Fishler and R.C. Boles: "Random sample consensus: A paradigm for model fitting with applications to image analysis and automated cartography", Communications of the ACM, Vol.24, No.6, pp.381-395 (1981)

(30) R. Hartley and A. Zisserman: "Multiple view geometry in computer vision, second edition", Cambridge University Press (2003)

(31) M. Grabner, H. Grabner, and H. Bischof: "Learning features for tracking", IEEE Conf. on Computer Vision and Pattern Recognition, pp.1-8 (2007)

(32) S. Cheng and C. Hu: "Particle filter tracking algorithm based on multiinformation fusion", Int. Conf. on Information Engineering and Computer Science, pp.1-4 (2009)

(33) PETS2001Dataset: ftp://ftp.cs.rdg.ac.uk/pub/PETS2001/DATA-SET1/

(34) PETS2000dataset: ftp://ftp.cs.rdg.ac.uk/pub/PETS2000/testimages/

(35) D. Doermann and D. Mihalcik: "Tools and techniques for video performance evaluation", Proc. IEEE Int. Conf. on Pattern Recognition, Vol.4, pp.167-170 (2000)

Xiaofeng Lu (Student Member) received the B.S. and M.S. degrees

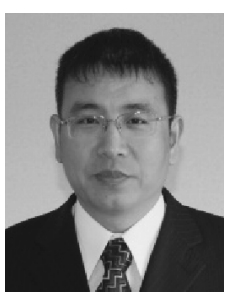
in computer science and technology from Xi' an University of Technology, Xi'an, China, in 2001 and in 2006, respectively. Now he is working towards the Ph.D. degree at Graduate School of Science and Technology, Nihon University, Japan. His research interests include image processing, object tracking and recognition. Currently, he is working on a research project on algorithms for vehicle detection and tracking in Intelligent Transport Systems. 
Takashi Izumi (Member) received the M.S. degree in Electrical En-

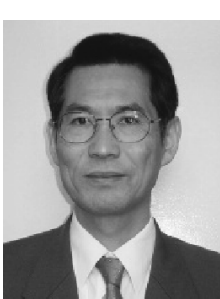
gineering from Nihon University in 1978. In 1978 he joined Department of Electronics as research associate, Nihon University. Presently, he is now a professor of Department of Electronics and Computer Science, Nihon University. He is Dr. of Eng. His research fields are related with route problem, image processing and computer science application to the traffic systems, etc.

Lin Teng (Student Member) received M.Sc. degree in Computer Sci-

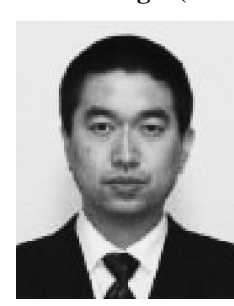
ence from Graduate School of Science and Technology of Nihon University in 2008. Now he is a Ph.D. student of Computer Science from Graduate School of Science and Technology of Nihon University.
Lei Wang (Non-member) received the B.S. and M.S. degrees in au-

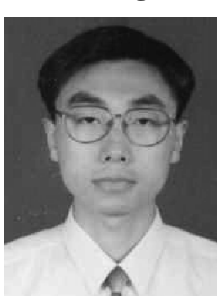
tomation engineering from Xi' an University of Technology, Xi'an, China, in 1994 and in 1997 respectively, and Ph.D. degrees from Xidian University, Xi'an, China, in 2001. During 2002 to 2004, he was a post-doc in the Lab of Pervasive and Artificial Intelligence at the Department of Informatics of the University of Fribourg (Switzerland). Since 2005 he has been with the School of Computer Science and Engineering Xi'an University of Technology, Xi'an,

China, where he became a Full Professor. His current research interests include signal and image processing, nonlinear circuits and systems theory, learning theory and algorithms, optimization problems, wavelet theory, and data mining. 